\title{
Improving trial design in cancer anesthesia (onco-anesthesia) research
}

\author{
Jonathan G. Hiller, MBBS, GCEpi, FANZCA · Paul S. Myles, MPH • \\ Rob G. Ramsay, PhD · Bernhard J. Riedel, PhD
}

Received: 1 March 2017/Revised: 8 March 2017/Accepted: 24 March 2017/Published online: 30 March 2017

(C) Canadian Anesthesiologists' Society 2017

\section{To the Editor,}

Adrenergic and inflammatory pathways are increasingly implicated in cancer progression and, because of their perioperative up-regulation, are hypothesized to have an adverse effect on long-term postoperative outcomes. ${ }^{1}$ Our recent prospective, randomized trial investigated whether perioperative celecoxib modified the inflammatory response following major intra-cavity cancer surgery. ${ }^{2}$ The primary endpoint of the study was prostaglandin $\mathrm{E}$ metabolite $\left(\mathrm{PGE}_{\mathrm{M}}\right)$, a biomarker of cyclooxygenase activity. The methodology, results, and analytical techniques may be found in the original manuscript. ${ }^{2}$ Here, we report a post hoc analysis examining the influence of neoadjuvant chemotherapy (NCT), administered within three months preoperatively, on patients' preoperative inflammatory state and on celecoxib's impaired effectiveness perioperatively. These findings provide

Registration Trial Registry Number: ACTRN12615000041550; anzctr.org.au

J. G. Hiller, MBBS, GCEpi, FANZCA $(\bowtie)$ · B. J. Riedel, PhD

Department of Cancer Anaesthesia, Perioperative and Pain

Medicine, Peter MacCallum Cancer Centre, Melbourne,

Australia

e-mail: jonathan.hiller@petermac.org

J. G. Hiller, MBBS, GCEpi, FANZCA - R. G. Ramsay, PhD Differentiation and Transcription Laboratory, Peter MacCallum Cancer Centre, Melbourne, Australia

J. G. Hiller, MBBS, GCEpi, FANZCA · B. J. Riedel, PhD Faculty of Medicine, Dentistry and Health Sciences, University of Melbourne, Melbourne, Australia

J. G. Hiller, MBBS, GCEpi, FANZCA · P. S. Myles, MPH Faculty of Medicine, Nursing and Health Sciences, Monash University, Clayton, Melbourne, Australia separate learning points with respect to the trial design and analysis in onco-anesthesia research.

Patients undergoing NCT $(n=10)$ compared with NCT-naïve patients $(n=19)$ had an increased preoperative $\mathrm{PGE}_{\mathrm{M}}$ (median [interquartile range], 30.3 [25.3-33.6] vs 21.9 [17.3-26.1] $\mathrm{pg} \cdot \mathrm{mL}^{-1}$, respectively; between-group difference, $8.4 \mathrm{pg} \cdot \mathrm{mL}^{-1} ; 95 \%$ confidence interval, 3.15 to $12.6, P<0.003)$. This difference suggests that patients undergoing NCT are in a persistent preoperative pro-inflammatory state. Furthermore, the subgroup of NCT patients given perioperative celecoxib $(n=6)$ had persistently elevated postoperative PGE levels compared with those of the NCT-naive patients $(n=9$; $P=0.03)$. The NCT-naïve patients randomly assigned to celecoxib had smaller changes from baseline in prostaglandin levels at six, 24 and $48 \mathrm{hr}$ following surgical incision compared to NCT patients (six hr: -4.1 vs $7.7 \mathrm{pg} \cdot \mathrm{mL}^{-1}, P<0.001 ; 24 \mathrm{hr}:-6.3$ vs $5.5 \mathrm{pg} \cdot \mathrm{mL}^{-1}$, $P<0.001 ; 48$ hr: $\quad-6.7$ vs $\left.8.0 \mathrm{pg} \cdot \mathrm{mL}^{-1}, \quad P<0.03\right)$ (Figure).

These data likely reflect an inability of celecoxib (administered at standard dosage) to inhibit the inflammatory response to surgery following NCT. This observation is supported by reports that chemotherapy dysregulates the post-translational modification of cyclooxygenase $^{3}$ and leads to non-steroidal antiinflammatory drug (NSAID) resistance in patients on palliative chemotherapy regimens. ${ }^{4}$

The limitations of study size and post hoc analyses are appreciated, and over-interpretation of these data must be avoided. Nevertheless, we wish to convey that - given the escalation in research on the impact of anesthesiology techniques and adjuvants (e.g., NSAIDs, $\beta$-blockers) on long-term cancer outcomes - researchers designing future studies should not only consider American Joint 


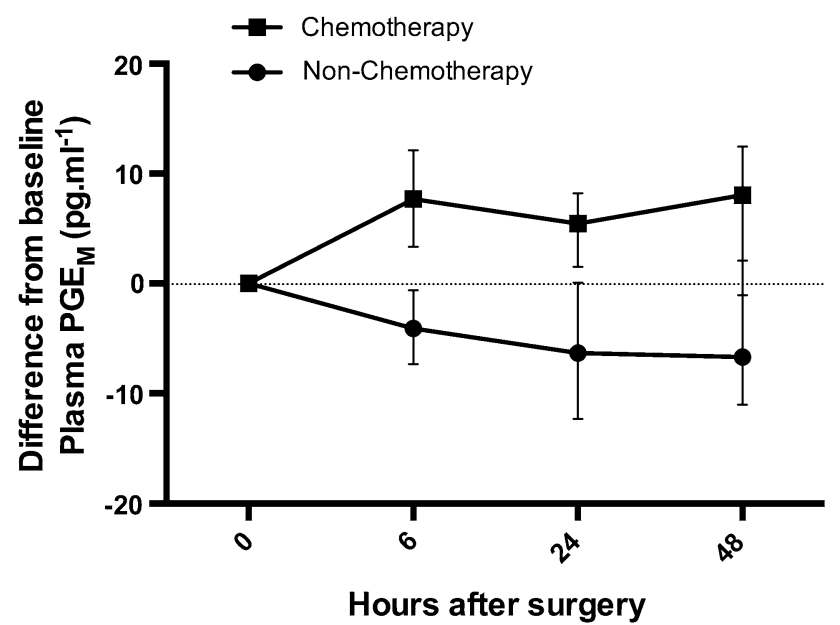

Figure Influence of neoadjuvant chemotherapy exposure on cyclooxygenase activity (prostaglandin $\mathrm{E}$ metabolite, $\mathrm{PGE}_{\mathrm{M}}$ ) in patients given perioperative celecoxib for major intra-cavity cancer surgery. Data (median, interquartile range) represent the change in $\mathrm{PGE}_{\mathrm{M}}$ from baseline to postoperative time points

Committee on Cancer specific tumour grading and lymphovascular space infiltration, ${ }^{5}$ but also the significance of NCT on the perioperative inflammatory response. Particularly, trials testing the effects of perioperative NSAID administration on cancer survival (e.g., NCT00502684, NCT00888797) should consider the currently under-emphasized NCT exposure in their analytical models. Future trials should also consider stratifying the randomization process based on whether trial participants underwent NCT.

Disclosures i) no external funding declared; ii) no conflicting commercial or non-commercial affiliations; iii) no competing interests declared.

Editorial responsibility This submission was handled by Dr. Philip M. Jones, Associate Editor, Canadian Journal of Anesthesia.

\section{References}

1. Byrne K, Levins KJ, Buggy DJ. Can anesthetic-analgesic technique during primary cancer surgery affect recurrence or metastasis? Can J Anesth 2016; 63: 184-92.

2. Hiller JG, Sampurno $S$, Millen $R$, et al. Impact of celecoxib on inflammation during cancer surgery: a randomized clinical trial. Can J Anesth 2017. DOI:10.1007/s12630-017-0818-z.

3. Dixon DA. Dysregulated post-transcriptional control of COX-2 gene expression in cancer. Curr Pharm Des 2004; 10: 635-46.

4. Reckamp KL, Krysan K, Morrow JD, et al. A phase I trial to determine the optimal biological dose of celecoxib when combined with erlotinib in advanced non-small cell lung cancer. Clin Cancer Res 2006; 12: 3381-8.

5. Hiller J, Ismail H, Riedel B. Improved quality of anesthesia and cancer recurrence studies. Anesth Analg 2014; 119: 751-2. 